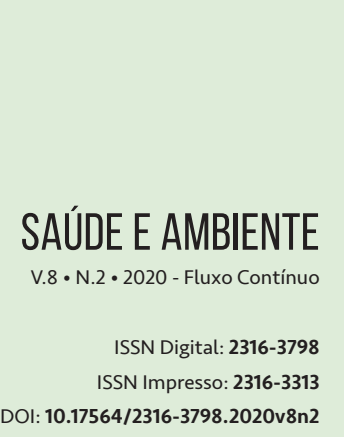

DOI: $10.17564 / 2316-3798.2020 v 8 n 2$
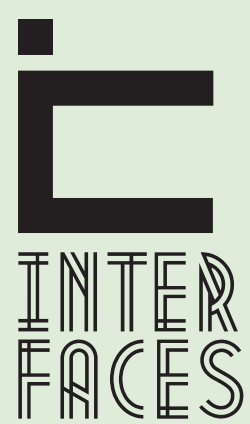

CIENTÍFICAS

\section{UNIVERSALIZAÇÃO E INTEGRALIDADE DO SERVIÇO PÚBLICO DE ESGOTAMENTO SANITÁRIO NO MUNICÍPIO DE SÃO LUÍS - MARANHÃO}

\author{
UNIVERSALIZATION AND INTEGRALITY OF THE \\ PUBLIC SANITARY SEWAGE SERVICE IN THE \\ MUNICIPALITY OF SÃO LUÍS - MARANHÃO
}

\section{UNIVERSALIZACIÓN E INTEGRALIDAD DEL SERVICIO PÚBLICO DE LA RED DE SANEAMIENTO EN EL MUNICIPIO DE SÃO LUÍS - MARANHÃO}

\title{
RESUMO
}

O saneamento básico é o conjunto de soluções relativas a abastecimento de água, disposição do esgoto sanitário, dos resíduos sólidos gerados e drenagem urbana. A Lei Federal $n^{0} 11.445$, de 05 de janeiro de 2007, determina as diretrizes e a concepção de saneamento básico e apresenta princípios particulares ao serviço público de saneamento básico, tais como: universalização do acesso; integralidade do serviço que são um dos grandes desafios do Brasil. Em função disto, este trabalho objetivou a análise do cenário atual do processo de universalização e integralidade do serviço de esgotamento sanitário no município de São Luís - MA. Para a concretização deste trabalho, foram feitas pesquisas bibliográficas, análise de dados de caráter qualitativo e quantitativo com base em dados do Sistema Nacional de Informações sobre Saneamento (SNIS) no período temporal de nove anos (2007 a 2016) e utilizou-se a Planta interativa de situação do sistema de esgotamento sanitário disponibilizado pela companhia de saneamento estadual. Diante do crescimento populacional na capital maranhense, notou-se 0 deterioramento da situação sanitária existente. Constatou-se algumas melhorias e os retrocessos que esses sistemas sofreram e o conflito entre o princípio da universalidade e da integralidade nas Bacias de esgotamento sanitário no Município.

\section{PALAVRAS-CHAVE}

Saneamento ambiental, Serviço público, Integralidade, São Luís. 


\section{ABSTRACT}

Basic sanitation is the set of solutions related to water supply, sanitary sewage disposal, solid waste generated and urban drainage. Federal Law No. 11,445, dated January 5, 2007, determines the guidelines and design of basic sanitation and presents particular principles to the basic sanitation public service, such as: universal access; integrality of service that are one of the great challenges of Brazil. This work aimed at analyzing the current scenario of the process of universalization and integrality of the sewage service in the city of. For the accomplishment of this work, bibliographical research was carried out, qualitative and quantitative data analysis from the National Sanitation Information System (SNIS) in the period of nine years (2007 to 2016) and the Interactive Situation Plan of the sanitary sewage system made available by state sanitation company was used. Faced with the population growth in the Maranhão capital, the deterioration of the existing sanitary situation was noted. There were some improvements and setbacks that these systems suffered and the conflict between the principle of universality and integrality in the Sanitary sewage basins in the Municipality.

\section{KEYWORDS}

Environmental Sanitation, Public Service, Integrality, São Luís.

\section{RESUMEN}

El saneamiento básico es el conjunto de soluciones relativas al abastecimiento de agua, disposición del alcantarillado sanitario, de los residuos sólidos generados y drenaje urbano. La Ley Federal $n^{0}$ 11.445, de 5 de enero de 2007, determina las directrices y la concepción de saneamiento básico y presenta principios particulares al servicio público de saneamiento básico, tales como: universalización del acceso; integridad del servicio que son uno de los grandes desafíos de Brasil. En función de esto, este trabajo objetivó el análisis del escenario actual del proceso de universalización e integralidad del servicio de alcantarillado sanitario en el municipio de São Luís - MA. Para la concreción de este trabajo, se realizaron investigaciones bibliográficas, análisis de datos de carácter cualitativo y cuantitativo con base en datos del Sistema Nacional de Información sobre Saneamiento (SNIS) en el período temporal de nueve años (2007 a 2016) y se utilizó la Planta de acuerdo con la normativa vigente. Ante el crecimiento poblacional en la capital de Maranhão, se notó el deterioro de la situación sanitaria existente. Se constataron algunas mejoras y los retrocesos que esos sistemas sufrieron y el conflicto entre el principio de la universalidad y de la integralidad en las cuencas de agotamiento sanitario en el Municipio. 


\section{PALABRAS CLAVE}

Saneamiento ambiental, Servicio público, Integridad, São Luís.

\section{INTRODUCÇÃO}

Nuvolari (2013), aponta o saneamento básico como o conjunto de soluções relativas a abastecimento de água, disposição do esgoto sanitário, dos resíduos sólidos gerados e drenagem urbana. No entanto, entende-se que o termo adequado e mais amplo é saneamento ambiental, compreendido como um conjunto de ações para preservar o meio ambiente e melhorar a saúde e a qualidade de vida da população.

Saneamento básico é, portanto, um conjunto de ações, serviços, infraestruturas e instalações operacionais de "todas as espécies de saneamento, quais sejam, o abastecimento de água potável; esgotamento sanitário; limpeza urbana e manejo de resíduos sólidos, drenagem e manejo das águas pluviais urbanas" (FREITAS, 2009, p. 908)

Segundo Craig (2000), os primeiros sistemas de esgotos foram construídos principalmente para afastar das cidades os resíduos produzidos pela população, o que era uma solução imediata que visava o controle. Tais práticas resultaram no cenário atual de esgotamento sanitário, pois a população preocupa-se apenas com o abastecimento e distribuição da água, então o uso e destino dela ficam à mercê.

A Lei Federal $n^{0}$ 11.445, de 5 de janeiro de 2007, determina as diretrizes e a concepção de saneamento básico e torna-se importante ao estabelecer o reconhecimento de subsídios para usufruidores que não possuem condições para custear o valor integral do serviço e a autonomia das instituições regulamentadoras do setor. Além disso, apresenta princípios particulares ao serviço público de saneamento básico, tais como:

Universalização do acesso;

Integralidade do serviço;

Adequação à saúde pública, ao meio ambiente, à segurança da vida e do patrimônio público e privado; Adoção de métodos, técnicas e processos que considerem as peculiaridades local e regional;

Articulação com políticas de desenvolvimento urbano e regional voltadas para a melhoria da qualidade de vida;

Utilização de tecnologias apropriadas, considerando a capacidade de pagamento dos usuários;

Transparência das ações;

Controle social;

Segurança, qualidade e regularidade;

Eficiência e sustentabilidade econômica e integração das infraestruturas e serviços com a gestão eficiente dos recursos hídricos (BRASIL, 2007).

Tais princípios, exigem grandes esforços entre as esferas de governo e os poderes públicos para a sua implementação bem como para garantir recursos a serem destinados à ampliação 
do atendimento à população. (SILVA, 2014). Tendo em vista que, o esgotamento sanitário é definido pela Lei do Saneamento (BRASIL, 2007) desde as ligações prediais até o seu lançamento final no meio ambiente.

A universalização e a integralidade dos serviços de saneamento básico, são um dos grandes desafios do Brasil. Segundo o Instituto Trata Brasil (2017), que divulgou um estudo com base em dados do Sistema Nacional de Informações sobre Saneamento (SNIS) de 2016 sobre serviços de saneamento mostra que apenas $12,1 \%$ dos maranhenses têm acesso aos serviços de esgotamento sanitário.

No ranking das 100 maiores cidades do país, São Luís-MA é a 79a no avanço do esgotamento sanitário segundo o Instituto Trata Brasil (2017). Embora ofereça serviços d'água tratada a cerca de 88,02\% da população, somente $4,03 \%$ do esgoto produzido é tratado e $47,9 \%$ da população tem acesso à coleta de esgoto (OLIVEIRA et al., 2016).

Em função disso, este trabalho teve como objetivo analisar o cenário atual do processo de universalização e integralidade do serviço de esgotamento sanitário no município de São Luís-MA.

\section{MÉTODOS}

A Ilha de São Luís compreende a microrregião da aglomeração urbana de São Luís e faz parte da mesorregião norte maranhense, abrigando em seu território quatro municípios: São Luís, Paço do Lumiar, São José de Ribamar e Raposa (SÃO LUÍS, 2011).

O município de São Luís está localizado na porção ocidental da Ilha, entre os rios Bacanga e Anil. De acordo com o último censo demográfico realizado pelo Instituto Brasileiro de Geografia e Estatística (IBGE, 2010), a capital São Luís possuía um total de 1.014.837 habitantes, com estimativa de 1.091.868 habitantes para 2017. O município apresenta importantes bacias hidrográficas como as dos rios Anil, Bacanga, Tibiri e Paciência.

A pesquisa e a análise de dados de caráter qualitativo e quantitativo foram realizadas a partir do Diagnóstico dos Serviços de Água e Esgoto, com base em dados do Sistema Nacional de Informações sobre Saneamento (SNIS). A busca foi feita no período temporal de nove anos (2007 a 2016), ou seja, desde que foi sancionada a Lei do Saneamento Básico até o último diagnóstico feito pelo SNIS, comparando três parâmetros: índice de coleta de esgoto, índice de tratamento de esgoto, o índice de esgoto tratado referido a água consumida e extensão da rede de esgoto por ligação ( $\mathrm{m} / \mathrm{lig}$ ).

Os dados coletados foram avaliados pela estatística descritiva, por meio de gráficos da série temporal de 10 anos, os quais apontam o avanço no serviço de esgotamento sanitário no Município de São Luís-MA. Para a identificação das zonas com maior déficit nos serviços de saneamento no município com abrangência da companhia de estadual de saneamento, utilizou-se a planta de situação do sistema de esgotamento sanitário (FIGURA 1). 


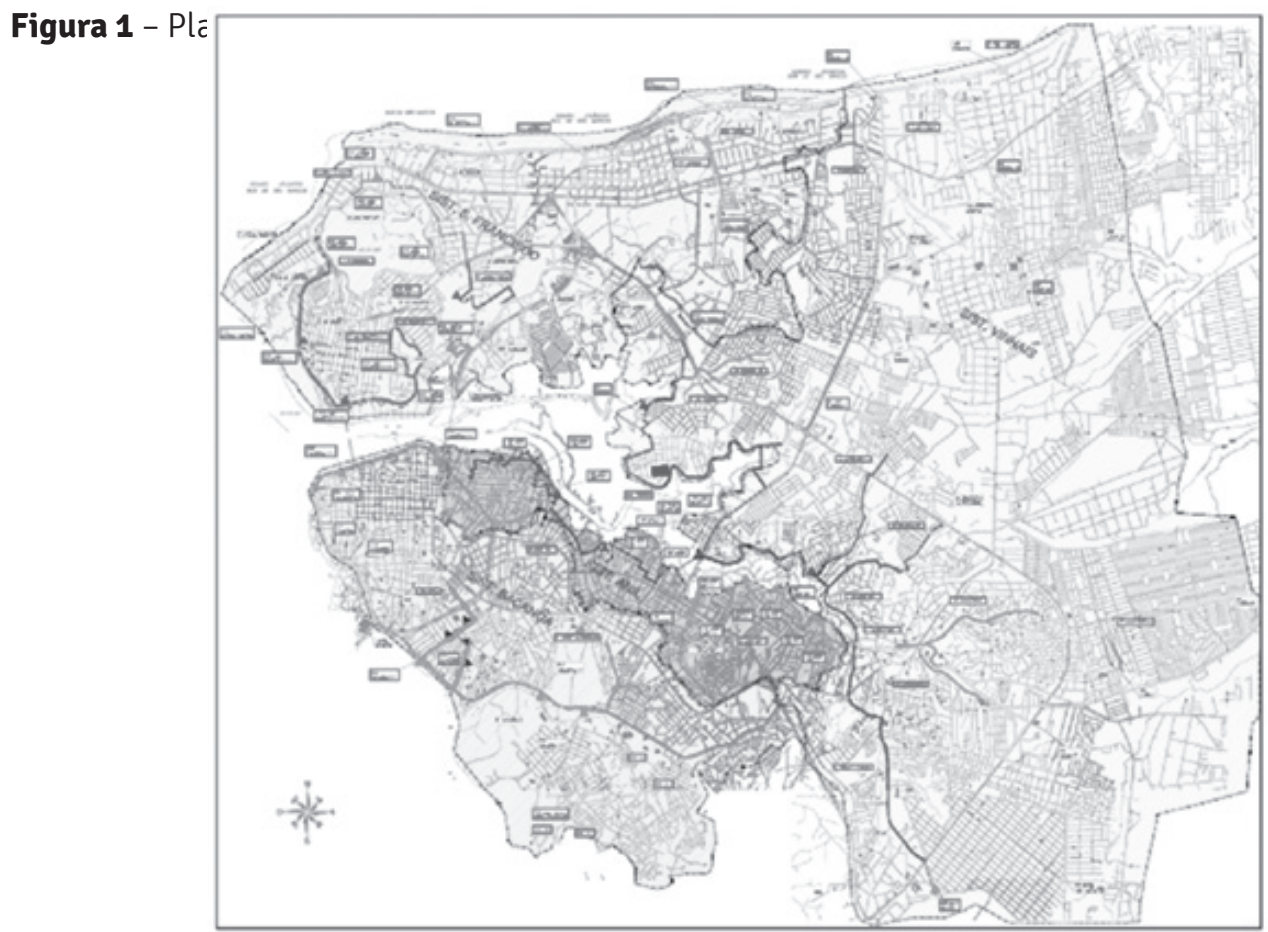

Fonte: CAEMA (2017).

\section{RESULTADOS}

Em São Luís-MA, a companhia de saneamento estadual é responsável pelos serviços de abastecimento de água e esgotamento sanitário. Além desta empresa, outro responsável também por uma parcela dos serviços de abastecimento de água, esgotamento sanitário e dos serviços de drenagem, é a Prefeitura Municipal de São Luís-MA, por meio da Secretaria Municipal de Obras e Serviços Públicos (SEMOSP), ambas são subordinadas à Superintendência de Saneamento Básico (SUSAN).

\subsection{IDENTIFICACÃO DAS ZONAS URBANAS DE ABRANGÊNCIA DA COMPANHIA ESTADUAL DE SANEAMENTO COM DÉFICIT NOS SERVIÇOS DE SANEAMENTO NO MUNICÍPIO}

No Município de São Luís-MA, o sistema de esgotamento é do tipo separador absoluto (ANJOS NETO, 2006). 0 sistema é dividido em quatro bacias de esgotamento sanitário, relacionadas às bacias hidrográficas da cidade, que são diretamente as principais afetadas pelos sistemas, tanto positivamente, quanto negativamente, uma vez que a maioria dos dejetos são lançados em seus corpos hídricos. 


\subsection{SISTEMA ANIL}

De acordo a companhia de saneamento estadual, a bacia do rio Anil possui 23.424 ramais prediais implantados e $265,89 \mathrm{~km}$ de rede coletora, que equivalem a 11,35 metros de rede por ramal predial. Divide-se em 56 sub-bacias de esgotamento, contendo, atualmente, os esgotos lançados diretamente no próprio rio Anil, canais da rede de drenagem em regiões alagadas, em seus afluentes e manguezais (IMESC, 2011).

O sistema de esgotamento sanitário do Anil está em expansão, sendo que possui uma Estação de Tratamento de Esgoto (ETE) em construção, 11 Estações Elevatórias de Esgoto (EEE) em execução com seus respectivos interceptores e linhas de recalque, como: EEE Cohab, EEE Porto das Dunas, EEE Ipem São Cristóvão, entre outras. Cerca de R\$24.621.808,68 foram investidos e alcançará aproximadamente 56 mil pessoas.

\subsection{SISTEMA BACANGA}

Segundo a companhia de saneamento estadual, o Sistema Bacanga conta com 106,00 km de redes coletoras. No entanto, apenas uma ETE fazia tratamento dos efluentes antes do descarte.

Atualmente, o Sistema Bacanga conta com uma Estação de Tratamento de Esgoto, quatro EEE. Foram investidos cerca de R\$ 8 milhões beneficiando 295 mil pessoas.

Verificou-se neste estudo que o número de Estações Elevatórias existentes (nove) é bem maior que as Estações Elevatórias em execução que chega apenas cinco, como EEE Bacanga 2.1.

\subsection{SISTEMA SÃO FRANCISCO}

O Sistema São Francisco possui 113 sub-bacias em uma área de $19 \mathrm{~km}^{2}$ que compreende parte da margem direita do rio Anil desde a ponte Bandeira Tribuzzi até a Bacia Oceânica. Tem a maior parte dos seus esgotos lançados no rio Anil, em regiões alagadas, manguezais e canais da rede de drenagem, sendo que parcela do esgoto coletado é tratado na ETE Jaracati.

O Sistema São Francisco possui uma Estação de Tratamento de Esgoto, 14 estações Elevatórias, quatro Estações Elevatórias em execução e sendo o único que possui 4 EEE em fase de licitação. Foram investidos R\$ 8,2 milhões e alcança 115 mil habitantes e recebeu investimentos do PAC2.

\subsection{SISTEMA VINHAIS}

O sistema comparado com os demais é o maior, dispondo de uma Estação de Tratamento, 14 estações elevatórias de esgoto, como: EEE do Ipem e Cohab. Além disso possui nove Estações Elevatórias em fase de execução, tais como: EEE Cohafuma e Cohajap. Foram investidos R\$ 63.439.496,64 e recebeu investimentos do PAC1 e PAC2. 


\subsection{ANÁLISE DA EFETIVAÇ̃̃O DOS PRINCíPIOS DA UNIVERSALIZAÇ̃̃O E INTEGRALIDADE NAS BACIAS DE ESGOTAMENTO SANITÁRIO NO MUNIĆ́PIO PREVISTOS NA LEI NACIONAL DE SANEAMENTO BÁSICO}

Atualmente o Município de São Luís-MA, opera com aproximadamente $71 \mathrm{EEE}$, que dão apoio aos três grandes equipamentos para tratamento do esgoto coletado nas ETE Vinhais, Bacanga e Jaracati. A ETE do Sistema Anil não foi entregue, as obras ainda estão em andamento.

Estas bacias de esgotamento sanitário apresentam índice de coleta de esgoto bem variável ao longo dos anos. Na Figura 2, destaca-se o índice de maior porcentagem referente ao ano de 2008, aumento de $108 \%$ referentes ao ano anterior, sendo que nesse período o Município contava apenas com duas ETE, Jaracaty e Bacanga. Esse aumento deve-se ao crescimento populacional.

Já o menor índice encontra-se no período de 2010, 2011 e 2012, devido a reparos nas Estações de Tratamento no Jaracaty e Bacanga que consequentemente resultaram na diminuição da coleta. Já nos anos de 2014, 2015 e 2016, com três sistemas de esgotamento em pleno funcionamento, constata-se menor variação no índice, resultado do crescimento de moradias, destacando-se os condomínios que possuem sua coleta de esgoto privada.

Figura 2 - Índice de coleta de esgoto (\%) do Município de São Luís

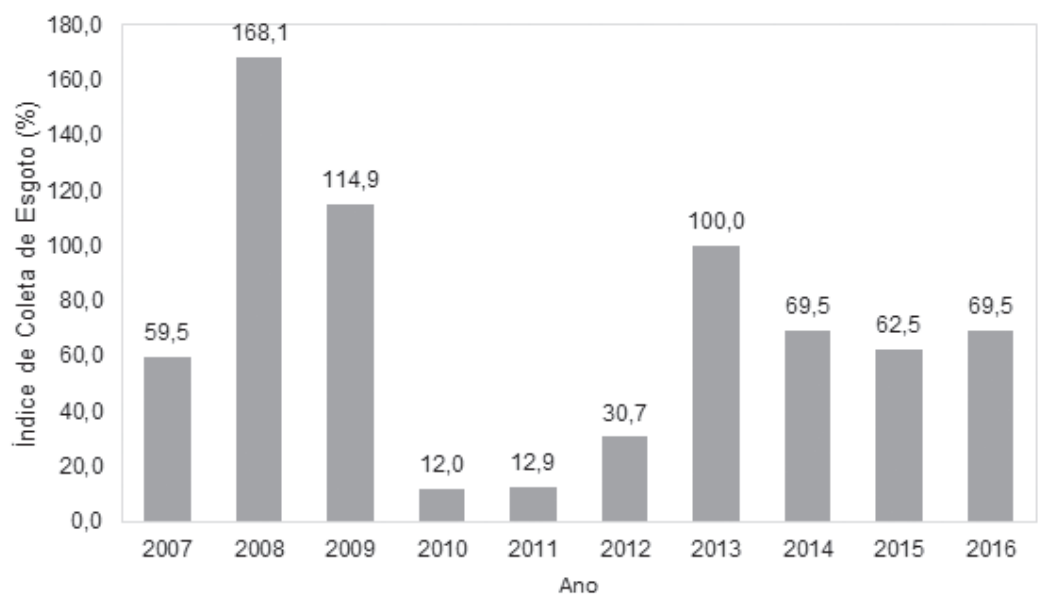

Fonte: SNIS (2016).

A deficiência de modo geral no tratamento de esgoto também é evidente no município de São Luís, que no período de 2013 teve seu menor índice durante os nove anos de estudo, chegando a $7,86 \%$ e cujo índice de coleta foi de $100 \%$, ou seja, mostra que não existe a universalização e a integralidade dos serviços, pois com o crescimento da população ocorre aumento na coleta, que resulta no aumento de dejetos a tratar. Tal situação torna-se de melhor compreensão nos anos a seguir, 2014, 2015 e 2016 (FIGURA 3). 
Figura 3 - Índice de tratamento de esgoto (\%) de São Luís

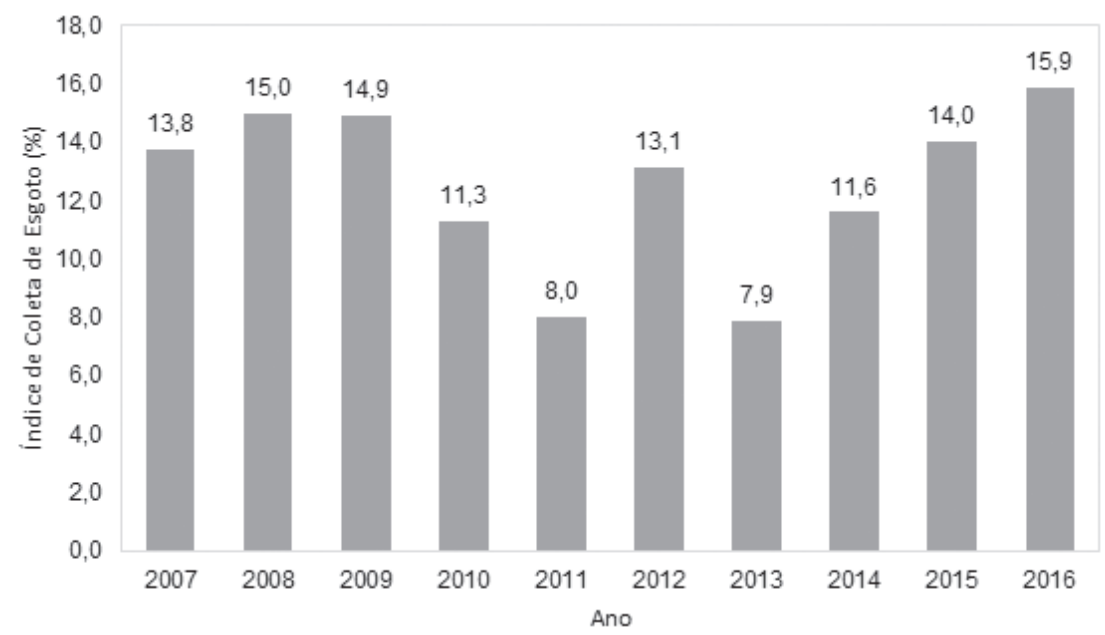

Fonte: SNIS (2016).

O crescimento no tratamento de esgoto referido a água consumida mostra que os números evidenciam um crescente avanço, apesar do tratamento de esgoto mostrar-se mais longe da universalização, devido a carência do setor e as necessidades de medidas e ações para que esse índice tenha uma melhora significante (FIGURA 4).

Figura 4 - Índice de esgoto tratado referido a água consumida do município de São Luís

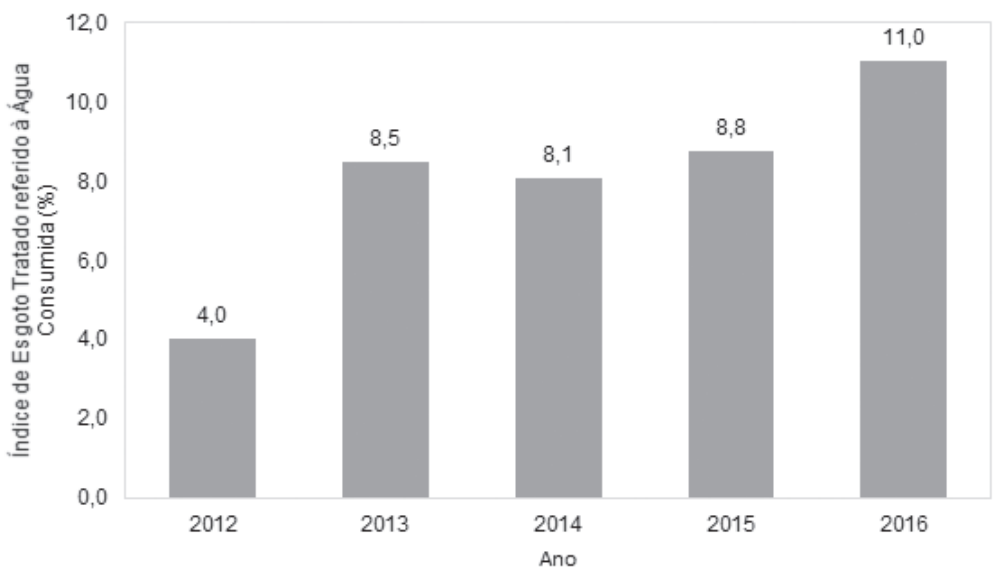

Fonte: SNIS (2016). 
Com relação ao avanço percentual da rede coletora de esgoto no Município, tendo em vista que nos anos de 2010 e 2011 não foram encontrados dados no SNIS (FIGURA 5).

Figura 5 - Extensão da rede de esgoto por ligação (m/lig)

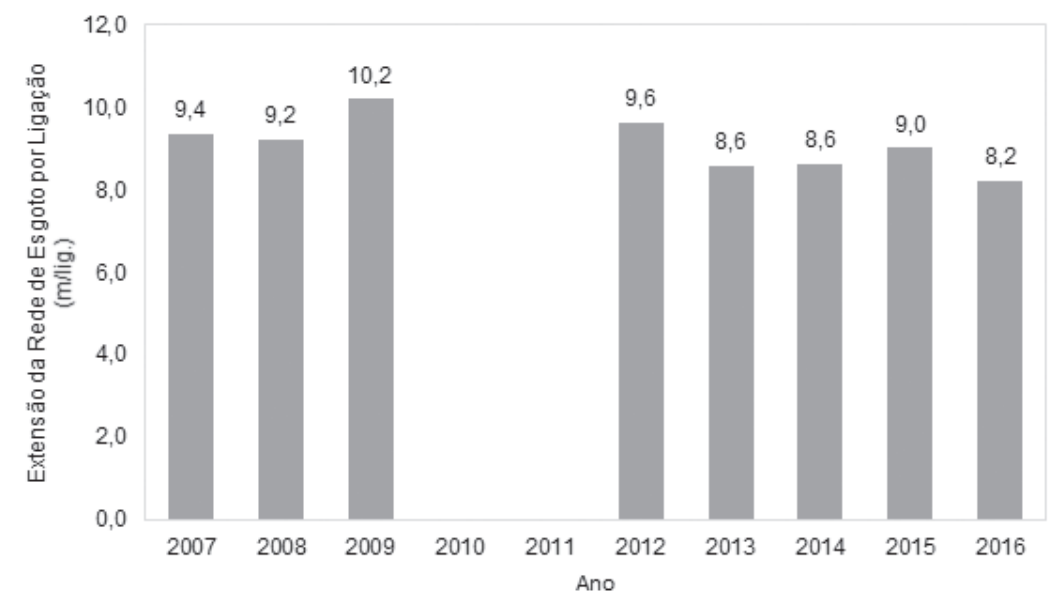

Fonte: SNIS (2016).

\section{DISCUSSÃO}

Na capital maranhense, diante ao crescimento populacional, notou-se o deterioramento da atual situação sanitária ao longo de muitos anos. A partir das deficiências do sistema de saneamento, a companhia estadual de saneamento juntamente com parceria com empresa privada desenvolveu no período de 1995 a 1998 estudos e projetos que foram nomeados "Programa de Saneamento Ambiental da Ilha de São Luís”, com objetivo de melhorar as condições sanitárias da época e futuras (ANJOS NETO, 2006).

O projeto teve abrangência maior na Ilha de São Luís e, conforme a companhia de saneamento estadual, teve como objetivos principais: a ampliação da rede coletora, de interceptores e dos emissários; o tratamento dos esgotos; a desinfecção dos efluentes; soluções econômicas, sanitária e ambiental; captação de recursos para dar suporte ao Programa de Saneamento Ambiental da Ilha de São Luís (ANJOS NETO, 2006).

O governo previa a partir desse projeto aumentar significativamente o índice de esgoto tratado na capital de $4 \%$ para $70 \%$ até 2018 e os investimentos já somam mais de 320 milhões de reais na ampliação do sistema de esgotamento sanitário no Município de São Luís-MA (PDITS, 2014).

Segundo a Organização Mundial da Saúde (OMS), a cada R\$ 1,00 investido em saneamento, R\$ 4,00 são economizados em saúde pública, sendo assim São Luís teria ganhos econômicos, sociais e ambientais com tais investimentos (INSTITUTO TRATA BRASIL, 2017). 
Nessa perspectiva, as bacias de esgotamento sanitário do Anil e Vinhais receberam verbas do Programa de Aceleração do Crescimento (PAC 1 e PAC 2). E as bacias do sistema Bacanga e São Francisco receberam verbas do programa PAC 2 (CAEMA, 2017).

No entanto, constatou-se alguns retrocessos e melhorias no sistema de esgotamento sanitário de São Luís ao longo dos anos. Verificou-se em primeiro momento um conflito entre o princípio da universalidade e da integralidade nas bacias de esgotamento sanitário no município, sendo que ambos princípios necessitam estar entrelaçados.

Assim, não basta o reconhecimento da universalidade, é necessário que haja a sua totalidade de serviços. Isto foi evidente quando se verificou que o tratamento não acompanha o índice de coleta de esgotos no município. Ainda existem estações sem funcionamento no município, como a ETE Anil e as outras não tratam esgoto conforme sua capacidade em função da falta de rede coletora que transporte o efluente gerado na cidade até estas estações.

No entanto, alguns avanços aconteceram, pois foi entregue em 2016 a ETE do Vinhais e vários interceptores e redes coletoras de esgotos foram instalados em São Luís, apresentando um grande ganho no município. Embora seja perceptível tais avanços, tardiamente, atualmente metade da população recebe os serviços prestados pela companhia estadual. 0 número total de habitantes é aproximadamente de 1.091 .868 e cerca de 519.175 habitantes são atendidos com esgotamento sanitário (SNIS, 2016).

No entanto, essas deficiências do sistema de esgotamento sanitário de São Luís com relação aos princípios da integralidade e universalização preconizados pela Lei 11.445/2007 trazem efeitos negativos para o meio ambiente e para a socioeconomia local. Porém, este é um cenário não apenas do presente município estudado, mas de muitos municípios brasileiros, gerando problemas ambientais devido ao lançamento de esgotos de forma indiscriminada em rios e mares.

\section{CONCLUSÃO}

Constatou-se a importância do saneamento básico em particular o esgotamento sanitário nos âmbitos social, ambiental e econômico. Apesar do desempenho positivo nos avanços na área de saneamento básico principalmente na área de esgotamento sanitário e nas lacunas legais, a universalização do acesso aos serviços em determinadas localidades do Município de São Luís-MA enfrenta diversas limitações, uma vez que, a universalização não depende somente dos recursos, mas principalmente da execução e continuidade dos serviços.

Assim como a universalização, a integralidade dos serviços públicos de saneamento básico tem relação diretamente aos aspectos econômicos, com aspectos políticos e institucionais, por isso a importância do monitoramento constante e eficaz por parte dos órgãos fiscalizadores do meio ambiente.

Os recursos são escassos e as necessidades da população são infinitas. Com o crescimento da população, surge então divisões quanto aos serviços oferecidos, por um lado os que só dispõem somente de alguns dos serviços e por outro lado os que não dispõem de serviço algum. 
A universalização e integralidade do esgotamento sanitário, principalmente nos serviços de tratamento e coleta de esgoto no Município de São Luís-MA, faz-se necessário urgentemente.

\section{REFERENCIAS}

ANJOS NETO, S. P. Aspectos históricos e diagnóstico técnico operacional do sistema de esgotos sanitários de São Luís. São Luís: [s.n], 2006.

BRASIL. Fundação Nacional de Saúde. Manual de saneamento. 3. ed. $1^{\text {a }}$ reimpressão. Brasília: Fundação Nacional de Saúde, 2007.

BRASIL. Lei $n^{0} 11.445,5$ de janeiro de 2007. Estabelece diretrizes nacionais para o saneamento básico; altera as Leis nos 6.766, de 19 de dezembro de 1979, 8.036, de 11 de maio de 1990, 8.666, de 21 de junho de 1993, 8.987, de 13 de fevereiro de 1995; revoga a Lei no 6.528, de 11 de maio de 1978; e dá outras providências. Diário Oficial [da] República Federativa do Brasil, Brasília, DF, 11 jan. 2007. Disponível em: www.planalto.gov.br/ccivil_03/_ato2007-2010/2007/lei/l11445.htm. Acesso em: 8 mar. 2018.

CAEMA - Companhia de Saneamento Ambiental do Maranhão. Planta das bacias de esgotamento sanitário do município de São Luís - MA. 2017

CRAIG, A. Overcoming expertocracy through sustainable development: the case of wastewater. In: IAPS Conference, 16 jul. 2000, Paris. 21st century: Cities, social life and sustainable development. Paris: [s.n.], [200-].

FREITAS, R. V. O marco regulatório do saneamento básico e a defesa do meio ambiente. Bol. Dir. Admin., v. 25, n. 8, p. 902-916, 2009.

IBGE - Instituto Brasileiro de Geografia e Estatística. Pesquisa nacional de saneamento básico 2008. Rio de Janeiro: IBGE, 2010. Disponível em: http://www.ibge.gov.br/home/estatistica/ populacao/condicaodevida/pnsb/pnsb.pdf.

Acesso em: 1 abr. 2018.

IMESC - Instituto Maranhense de Estudos Socioeconômicos e Cartográficos. Situação Ambiental da Itha do Maranhão. São Luís: IMESC, 2011.

INSTITUTO TRATA BRASIL. Saneamento e Saúde. 2017. Disponível em: http://www.tratabrasil.org. br/saneamento/principais-estatisticas/no-brasil/saude. Acesso em: 18 mar. 2018. 
NUVOLARI, A. Dicionário de saneamento ambiental. São Paulo: Oficina de Textos, 2013.

OLIVEIRA, G. et al. Ranking do Saneamento. Resultados com base no SNIS 2016. São Paulo:

Instituto Trata Brasil, 2016.

SÃO LUÍS. Secretaria Municipal Extraordinária de Projetos Especiais. Plano municipal integrado de saneamento básico PMISB de São Luís - MA (2011). Disponível em: http://www2.saoluis.ma.gov. br/custom_files/File/Arquivos\%20Pref\%2003_06_2013/Produto_II_Diagnostico.pdf. Acesso em: 2 jun. 2018.

SILVA, G.S. et al. Avaliação integrada da qualidade de águas superficiais: grau de trofia e proteção da vida aquática nos rios Anil e Bacanga, São Luís (MA). Rev. Eng. Sanit. Amb., v.19, n. 3, p. 245-251, 2014. Disponível em: http://www.scielo.br/scielo.php?pid=S1413$41522014000300245 \&$ script=sci_arttext. Acesso em: 2 abr. 2018.

SNIS - Sistema Nacional de Informações sobre Saneamento. Ministério das Cidades. Diagnóstico dos serviços de água e esgotos - 2016. Disponível em: http://www.snis.gov.br/diagnostico-anualagua-e-esgotos/diagnostico-ae-2016. Acesso em: 6 mar. 2018. 
1 Engenheira Ambiental. E-mail: mahelliad@gmail.com

2 Acadêmico em Engenharia Ambiental, Universidade Ceuma. E-mail: manoelneto96@hotmail.com

3 Acadêmica em Engenharia Ambiental, Universidade Ceuma. E-mail: larissa.nogueiravieira@hotmail.com

4 Acadêmica em Engenharia Ambiental, Universidade Ceuma. São Luís. Maranhão. Brasil.

E-mail: pollyanna.pedrosa@hotmail.com

5 Acadêmica em Engenharia Ambiental, Universidade Ceuma. E-mail: thalissa1949@hotmail.com

6 Acadêmico em Engenharia Ambiental. Universidade Ceuma. São Luís. Maranhão. Brasil.

E-mail: moisessantos120@gmail.com

7 Especialista em Perícia e Auditoria Ambiental e em Gestão de Recursos Hídricos; Engenheiro Ambiental, Companhia de Saneamento Ambiental do Maranhão.

E-mail: rengawwg@hotmail.com

8 Mestrado em Sustentabilidade de Ecossistemas; Professora, Universidade Ceuma; Engenheira Ambiental.

E-mail: prof.nathaliapinheiro@gmail.com

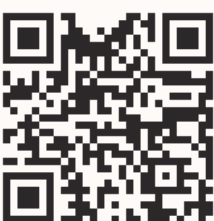

A autenticidade desse artigo pode ser conferida no site https://periodicos. set.edu.br

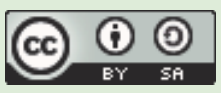

Este artigo é licenciado na modalidade acesso abertosob a Atribuição-Compartilhalgual CC BY-SA

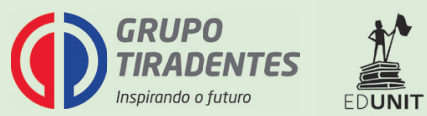


